\title{
Effective Dynamic Range Evaluation of Digital Still Images in Mobile Devices
}

\author{
Irma PUŠKAREVIĆ, Uroš NEDELJKOVIĆ, Dragoljub NOVAKOVIĆ, Ivan PINĆJER
}

\begin{abstract}
Digital photography has immersed in everyday surroundings through mobile devices which became the most popular tool for taking pictures. However, the compactness of these devices is an obstacle for certain image quality parameters when it comes to usability. The purpose of this paper is to analyze the effective dynamic range of mobile device cameras. We obtained opto-electric conversion function (OECF) measurements according to ISO standard for total and effective dynamic range evaluation. Additionally, the evaluation of effective dynamic range using high dynamic range image construction was performed where algorithms for tone mapping were applied. Our findings indicate little difference in OECF values among different mobile device cameras, as well as for the total dynamic range values. However, when it comes to the effective dynamic range, cameras in these devices show less input-output conversion stability. Additionally, tone mapping indicates that the effective dynamic range is different between devices.
\end{abstract}

Keywords: digital imaging; dynamic range; image processing; mobile devices; tone mapping; objective metrics; OECF

\section{INTRODUCTION}

So far, usability assessment of image quality and advances in signal processing have been there to aid the work of professional and amateur photographers who use DSLR (digital single-lens reflex) cameras, and also to help develop their mass-produced compact version. Following the development of new technologies, the image capture system architecture of these cameras has been transferred to mobile phones and tablets. These hybrid devices have been commercially adopted faster than any other technology in modern history. The ubiquity of these devices contributed to the popularity of mobile photography for personal requirements but also for health and social services. Expectedly, the requirements of these users are high when it comes to the tone reproduction quality. The tone reproduction of a digital still image camera describes how the range of intensities in the original scene is mapped to a digital output image. This process is limited by several factors, one of which is the encoding of the dynamic range (DR) i.e. the range of the maximum and minimum measurable light intensities. Hence, the problem of tone reproduction in digital photography is closely related to reproducing high dynamic range scene on a media with limited dynamic range, such as a small screen of a mobile phone. The tone reproduction problem has been the subject of many research studies [1-5]. However, little research has focused on the tone reproduction quality of digital still images obtained with mobile device (MD) cameras.

The particularity of all digital image capture systems is a limited dynamic range in comparison to the human visual system (HVS). One of the most commonly used techniques for improving the dynamic range is shooting multiple photographs of the same scene with different exposure times i.e. high dynamic range imaging (HDRI) [6]. The content of HDR images justifies its steady popularity - images are visually more appealing and realistic, having the ability to bring an image closer to the dynamic range of the real world luminance. Even though dynamic range presents the maximum number of tonal steps that a device can discern, the full range of intensities is considered to have little relevance to the real-world photography. Regarded to have more relevance is the effective dynamic range, also referred to as the practical or useful range of recorded intensities. In relevance to mobile photography, we believe this feature has been neglected in research studies. With mobile devices (MD) becoming an everyday occurrence, due to their easy access to data, data transfer or data transmission, not only among professional or amateur photographers but health centres, artistic communities or development centres for mobile intelligence development of the accuracy of image capture system integrated into mobile devices can be more than beneficial $[7,8]$. Evaluating the effective dynamic range of digital still image capture system on MDs can help assess the technical quality of tone reproduction in this instance. Furthermore, expanded knowledge in this domain will aid future development of tone mapping algorithms. Drawing on conclusions from Urbano et al. [9] the aim of this study is to evaluate the quality of digital images by investigating the effective dynamic range of MD cameras and discuss main features at device-level.

\section{RELATED WORK}

The digital still-picture camera, being an integral part of the image capture system, has a task to reproduce the original scene [10]. Therefore, the theory of tone reproduction is described as the reproduction of physical values of light intensities [11]. It is difficult to provide a wholesome analysis of the performance of the digital stillpicture camera. There are several general characteristics of interest. However, a single function by the means of the single method cannot fully describe each of these characteristics. When testing mobile phone cameras, some characteristics are mandatory for the testing procedure, namely OECF and dynamic range [12]. We will discuss this matter further in the following section.

\subsection{Measuring the Characteristics of Digital Cameras}

One of the most significant measures of the quality check of digital still-picture cameras is OECF (optoelectronic conversion function). OECF describes the relationship between the illumination on the sensor and digital output values in the image i.e. an average digital 
response of the electronic device to light stimuli. In technical terms, the OECF curve is not as much a tone reproduction curve as it is a colour encoding curve which is dependent on several parameters: exposure speed, specific digital encoding of a particular camera, and additional tone values applied to an image (lightness/contrast, gamma correction) (Fig. 1) [13].
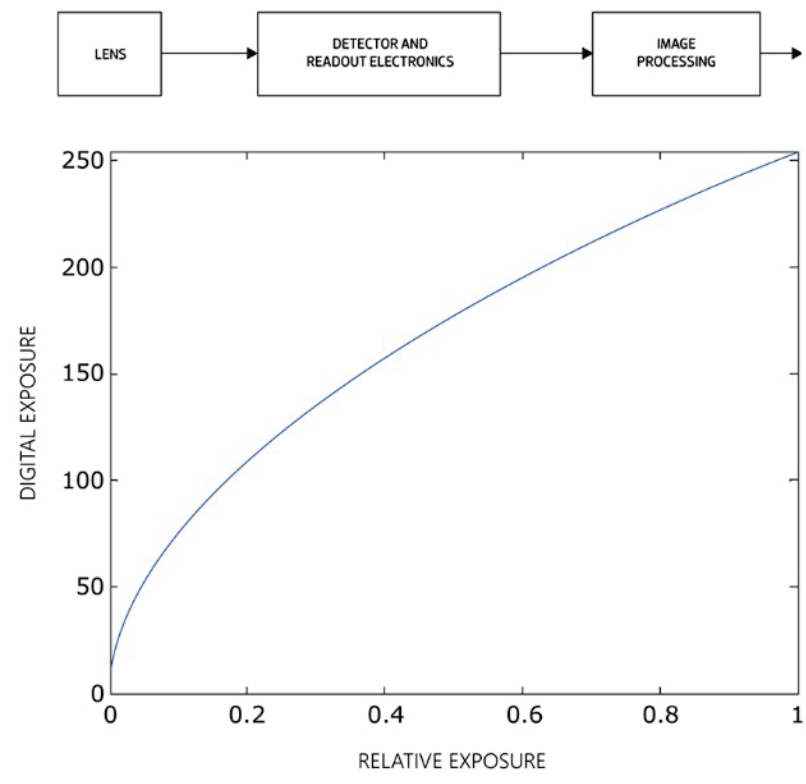

Figure 1 Elements of digital camera and a typical OECF curve that results from such signal transformation [13]

Test methods of camera OECFs are described in ISO 14524 [14] for both camera and focal plane OECFs. Repeated measures in such testing methods are possible for specific scene conditions such as taking pictures of a test chart under controlled conditions. The test chart for measuring camera OECF simulates the effect of the original scene luminance and average distribution of light intensities. For digital still-picture cameras that have interchangeable lenses, or a camera with a fixed lens but with an option to adjust exposure speed, the optical scene of image formation is not relocated, and the test chart which records the sufficient number of scene variations, is being translated to the camera sensor allowing OECF value to be analysed from the test chart image. The whole process is recorded with a single exposure unlike the measuring of the focal plane OECF (needed for cameras with fixed lenses such as compact or mobile phone cameras) which requires multiple exposures for accurate testing [15]. For the alternative focal plane measurement, the sensor luminance is calculated from the following equation:

$$
E_{\mathrm{s}}=\frac{0.65 L_{\mathrm{t}}}{f_{\mathrm{e}}^{2}}
$$

where $E_{\mathrm{s}}$ is the illuminance in lux falling on the sensor, $L_{\mathrm{t}}$ is the arithmetic mean luminance of the target in candela per square meter, and $f_{\mathrm{e}}$ is the effective $f$-number of the lens. According to Wüeller and Gabele [16], OECF can be used in a process of digital camera calibration as a function of measuring luminance. Additionally, in relation to the photographic process, OECF measurements are very important for images with HDR [17].

\subsection{Tone Mapping Operators and HDR Compression}

A tone mapping technique is able to generate images visually similar to a real scene i.e. tone mapping operator (TMO) is capable of significant contrast reduction to fit the displayable range [18-20]. In the attempt to develop accurate digital reproduction pipeline, a number of TMOs have been developed over the years [21-25]. The downside of TMOs is that they cause information loss, and the tone mapped content can have reduced perceptual quality since the DR compression tends to damage important details and textures. Further specific research is needed to develop TMOs particularly suitable for SSD, such that would emphasize certain features of the tone mapping process more effectively for these purposes [9].

TMOs are generally classified into two categories: spatially uniform (global) and spatially varying (local). Global operators $[1,26]$ use the same mapping function for the whole image, unlike local operators which employ a spatially varying mapping dependent on the local content $[22,27]$. The main advantage of global operators is the simplicity and the computational efficiency. However, their weakness lies in the impediment to keeping the appropriate local contrast in each region of the image. Local operators, on the other hand, are more flexible in controlling local contrast because the mapping technique is used for each pixel. As a result, the final image has a more pleasing effect since the human eye reacts locally to contrast [28]. However, local operators provide "halos" during contrast reversal. The most commonly used operators today $[23,25,29]$ apply logarithmic compression of the luminance values, dodging and burning techniques or decomposition of HDR still image. Therefore, the baseline behind HDR content compression is to reduce visual information from HDR to LDR device display so it is not noticeable by our eyes.

TMO is used for creating an LDR version of the HDR still image. During this compression, great attention is given to developing an algorithm that will preserve as much contrast as possible. DR and average luminance of the scene, present two important parameters in rendering performance of the algorithm, and are computed from (2) and (3):

$$
\begin{aligned}
& \text { Dynamic range }=\log _{2} L_{\mathrm{Max}}-\log _{2} L_{\mathrm{Min}} \\
& \text { Average Luminace }=\log _{2} \bar{L}_{w}-\log _{2} L_{\mathrm{Min}} \bar{L}
\end{aligned}
$$

Where $\bar{L}_{w}$ is the world luminance for pixel $(x, y)$. When analysing the DR of the camera, one discusses the total DR over which noise remains under a specific level. Besides the total DR analysis, it is necessary to take into consideration EDR (effective dynamic range) or practical DR. EDR is very important when considering the practical aspect of photography which refers to the perceptual quality of an image [30]. It describes how much detail can be drawn from the dark regions before the image noise becomes undesirable having in mind that DR generally is limited by noise. The balance between noise reduction and preserving details depends mostly on the noise received through the input signal. This particularly presents a problem for compact cameras and built-in cameras in mobile devices that work with limited signal information 
and considerable noise to begin with [31] For example, most of the DSLR cameras use 10 or 14-bit A/D converters which theoretically provide the overall DR of 10 to $14 \mathrm{f}$ stops, where $f$-stop is calculated from

$$
f-\text { stop }=\frac{\text { focal length }}{\text { aperature diameter }}
$$

When the processing noise is taken into consideration, we can actually count on 8 to 12 effective $f$-stops. The EDR can be improved by using multiple photographs of the same scene with different exposure times (HDR) [6]. According to Robertson et al. [6] the HDR output image contains a weighted average of the multiply-exposed input images, hence obtaining information captured by each of the input images. Disclosed indicates that TMOs are very important factor in the HDR rendering performance, given that they are the first step in a typical HDR content compression pipeline. It has also become a coding scheme, a widely adopted standard which supports almost all software and hardware equipment dealing with digital imaging, including MDs. However, MDs have limitations concerning hardware and software components that make it more difficult to apply HDR pipeline. Due to these limitations e.g. relatively poor quality of the sensor, a shutter (if present), aperture, optics or flash, we can expect optical aberrations and noise in a final digital image. The software limitations introduce additional problems because API usually prevents the access to the raw sensor data and often offers an interface that gives the JPEG compressed files. Nonetheless, Mantiuk et al. [32] show that HDR pipeline can be efficiently implemented in MDs proposing a pipeline architecture consistent with all the features of the standard HDR pipeline. The question arises how stable is the encoding on such platforms with the above-mentioned limitations and how much of usable DR can be expected? Methodology in this study uses algorithms for global and local tone mapping of commercially available software, as well as ISO 14524 for obtaining OECF values, in order to evaluate EDR of digital still images acquired with different MD cameras with regard to aiding the future development of rendering algorithms.

\section{METHODOLOGY}

In this section, we measure the tonal response (OECF) of mobile device cameras and analyse the effective dynamic range. We also study how the effective range of intensities is influenced by the tone mapping operators when used for HDR image acquisition.

\subsection{Details of the Objective Study}

Perceptual evaluation has been used in previous studies to test TMOs by comparing tone mapped image to a reference scene [33-36]. Even though experimentation with participants provides relevant insight, it may not always be the most appropriate approach, since the quantification of such test scenes is difficult to see [37]. The work described in this study concentrates primarily on objective metrics for evaluation of EDR, followed by the application of EDR concept to investigate the effectiveness of TMOs on digital still images generated with MDs. The Imatest program was used to obtain the key image quality factors in MD cameras discussed in Section 2. The program was used to compute tonal response (OECF) measurements for different density values of the test chart [38].

During DR analysis, the software, such as Imatest, computes several levels of DR depending on the noise threshold: 1.0 (low image quality), 0.5 (medium image quality), 0.25 (medium-high image quality) and 0.1 (high image quality). The DR is perceived as a difference between two zones of the two ends of contrast: the zone where the pixel level is $98 \%$ of its maximum value (250 out of 255 for 24-bit colour) and the darkest zone that meets measurement criterion. The noise factor is only meaningful in relation to a signal, hence the signal-to-noise ratio $(\mathrm{SNR}$ or $\mathrm{S} / \mathrm{N}$ ) is often calculated. When using a Stepchart, Imatest modules provide noise measurements in pixel levels or $f$-stops (or EV). Noise measured in $f$-stops corresponds closely to human vision $(1$ f-stop $(1 \mathrm{EV})=$ 0.301 density units; 1 density unit $=3.32$ f-stops $(\mathrm{EV})$.

\subsection{Testing Conditions}

Firstly, the experimental conditions were set up to obtain the opto-electronic conversion function as to test the camera's sensor reaction to changes in exposure. We used six MD cameras (three smartphones and three tablets), as well as one proconsumer DSLR camera which was taken as a reference device. Their available characteristics are presented in Tab. 1. The DR is measured by Stepchart using reflective Kodak Q-13 chart with a linear arrangement of grey patches (see Fig. 2). The test chart was illuminated in the lightbox Agile Radiant Controlled Lights with a simulation of the CIE standard illuminant D50. Following ISO 14524 proposition on lighting set-up, the room in the laboratory was dark as to secure no additional lighting goes through. The photographs of the test chart were shot with available focus points on the screen of the MD. The sharpness of the acquired images was in defined limits (some slightly out of focus) allowing tonal value detection between grey patches. The distance of the MD from the test chart was such that a camera lens could cover the length of the chart. The lens was placed vertically on the chart; grey patches were cantered in the shot to avoid vignetting. With the expected physical instability of MDs, all test devices were fixed to the surface. Each test device was used to make nine images with nine exposures as to ensure credible results and minimize the appearance of random noise in the input data at each exposition. The referenced DSLR camera (Device R) was subjected to the same procedure even though OECF measurements can be obtained with a single exposure in this case (ISO 14524).

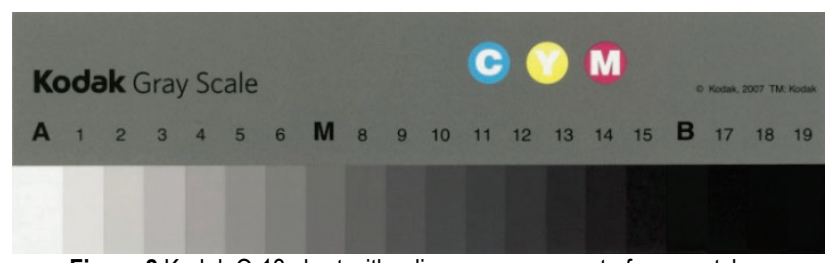

Figure 2 Kodak Q-13 chart with a linear arrangement of grey patches 


Table 1 Resolution and characteristics regarding photo settings for tested MD
\begin{tabular}{|c|c|c|c|c|c|c|}
\hline CAMERA & Device A & Device B & Device C & Device D & Device E & Device F \\
\hline RESOLUTION & $0.7 \mathrm{MP}$ & $5 \mathrm{MP}$ & $5 \mathrm{MP}$ & $8 \mathrm{MP}$ & $5 \mathrm{MP}$ & $8.7 \mathrm{MP}$ \\
\hline CHARACTERISTICS & & $\mathrm{AF}, \mathrm{EV}$ & $\mathrm{AF}, \mathrm{EV}$ & $\mathrm{AF}, \mathrm{OIS}, \mathrm{HDR}$ & $\mathrm{AF}, \mathrm{EV}$ & $\mathrm{AF}, \mathrm{OIS}, \mathrm{EV}$ \\
\hline
\end{tabular}

Generally, it is possible to apply one of the two approaches for camera settings [12]. The first approach implies the use of automatic settings, whereas the second approach suggests using the settings that will provide the optimal image quality. The settings available for MD cameras do not always include comprehensive manual settings that can be seen at DSLRs. Additionally, the manual exposure settings are not listed among mandatory settings on MDs.

For the purposes of this experimentation, it was necessary to set up all the test devices with options for manual settings through the installation of additional applications. Both Android and iOS platforms were equipped with equivalent applications (Camera FV-5; Camera + Pro). In the continuation of the study, HDR image construction and tone mapping procedure were applied. Each tested device was used to make three different exposures of the same scene, one short and one long exposure, and one with approximately average exposure of the two. Even though there is a possibility to integrated HDR setting on MDs, not all manufacturers integrate this option. Therefore, HDR construction was done differently for the purposes of this study. Assuming that MDs employ different processing at the input, we applied the same global and local operator on the output image. Consequently, we extracted original shots from MDs and processed them on the computer. A global and local TMO from the commercially available software Photomatix 5.0.5 was used. Once the HDR image was constructed it was saved as 8-bit JPEG file for further processing via Imatest program. In both procedures of the measurements, only the exposure time was altered.

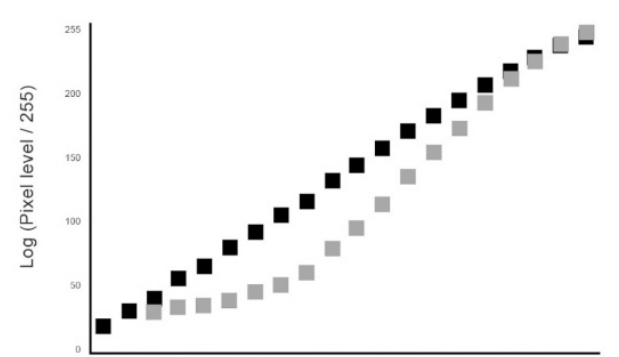

Log Exposure (Target Density - Device A)

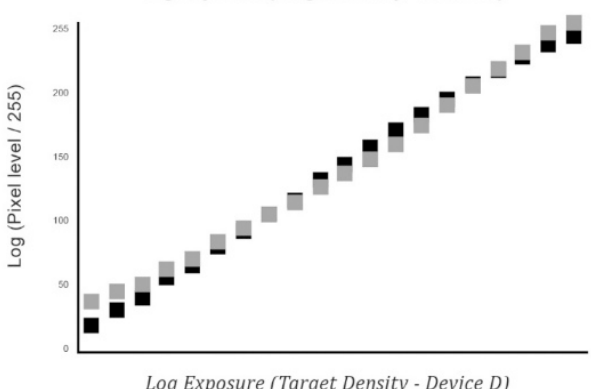

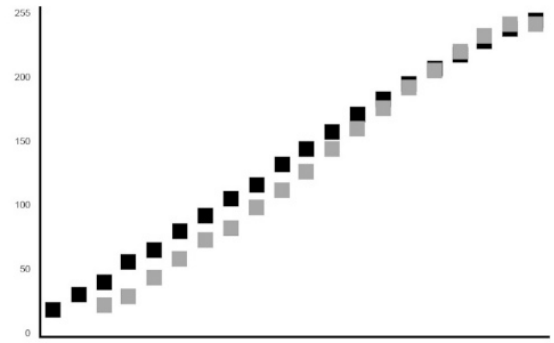

Log Exposure (Target Density - Device C)

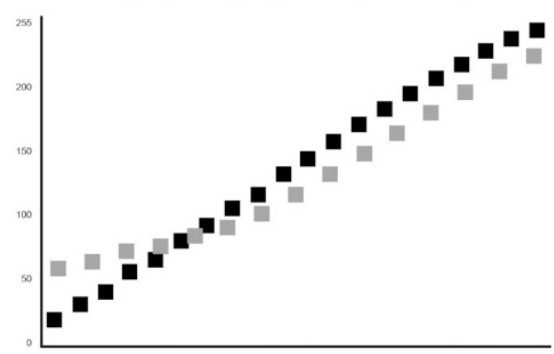

Log Exposure (Target Density - Device E)

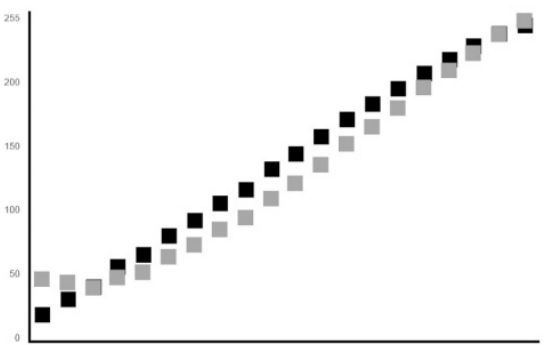

Log Exposure (Target Density - Device F)

Figure 3 A system response curve OEFC computed for MD cameras (grey patches), referenced to Device R curve (black patches)

\section{$4 \quad$ RESULTS AND DISCUSSION}

We tested 6 MD built-in cameras to evaluate the extent of the full range of usable intensities that can be encoded. The evaluation was performed using 8-bit JPEG images generated by API of the MD. Imatest software was used in order to obtain a comprehensive analysis of digital camera test images. Presented here are the obtained plots that relate to density response (OECF) curve, the DR of the camera and noise measurements. The lighting conditions were the same for all tested cameras. However, automatic settings were used to simulate real-scene utilisation. The minor limitation was the luminance conversion software which was different for each device. Since EXIF data was available for every image taken, we found that most common aperture ranges were between $\mathrm{f} / 2.0$ and $\mathrm{f} / 2.7$ and that ISO speed was between $32-100$.

\subsection{OECF and Effective Dynamic Range Analysis}

A system response curves i.e. the relationship between exposure values and pixel level in the form of OEFC function are observed in Fig. 3. Following explained methodology, we analysed the shape of the curve for the nine exposures of each camera. Even though there were slight shifts to the left or right across the exposure time, the transfer curve of the camera always had the similar OECF. From the curve shapes, it should be noted that there is no significant difference in the electronic gain between MD cameras and that the automatic conversion function increased the output digital level under the underexposure condition. The plots of Device E and F suggest the largest increase of the digital output level in the dark areas. All of the cameras did reach their saturation suggesting that these cameras maintain better conversion of input light for highlights than for shadows. There is a slight "shoulder" effect in some of the camera's transfer curve (most noticeable for Device C) which improves the image output 
by lowering the tendency of highlights to saturate. Notice how Device A shows a higher rate of deviation of the signal in shadows than other tested cameras. Presumably, the nonlinearity of the OECF curve is due to a gamma correction present in the image processing system of the camera. When compared to DSLR transfer curve, we can observe the greater nonlinearity of MD cameras.

Analysing the shape of the OECF curve of MD cameras we observed that the tested cameras showed a great increase of the digital output level in dark areas. This was expected due to the signal conversion which increases the digital output level for underexposed parts (see Section 2 ). When the conversion is more accurate more grey patches are discerned, suggesting that MD cameras still lack the appropriate intensities conversion stability. The output values suggest that each manufacturer uses their own algorithm for conversion. Unexpectedly, low sensitivity was detected for Device $\mathrm{E}$ which is among the newer models on the market. This can partly be explained by ISO speed which is set to 32. In a combination with a hand-held camera and low light conditions, it cannot yield satisfactory results. With automatic settings OECF curve describes adequate gradation for highlights, which corresponds more satisfactory with gamma conversion, unlike in the dark areas. Slight "shoulder" curve implies a computation that limits the images to "burn out" i.e. prevents the bright areas in the image to over saturate (pixel level 250 or 255). This is very important for the EDR and perceptual assessment of the image quality.

The difference in density values between zones, described as the total DR of the camera, is obtained by a reflective step chart. The reflective step chart is inadequate for testing DSLR cameras because it has a density range of 6.3 f-stops $(0.05-1.95)$ [39] which is noticeable in the readings of Device R. Most DSLR cameras have a DR over $10 f$-stops. However, reflective step charts can be used with multiple exposures and digital image capture system with lower DR, that is to say, it was sufficient enough for the testing in this study. The DR is determined by Imatest program which detects chart zones using the smallest density step that results in uniformly spaced detected zones. It can be observed from Tab. 2 that the detected patches in the case of MD cameras have a density range of 4.8 to $6.2 f$-stops, equalling a total DR of the camera. As this should not be interpreted as the camera's DR, greater significance to this study is the reading of high DR which will be interpreted as the usable density detected. For MD cameras, this reading is between 4.8 to 5.9 f-stops. It is noticeable how it decreases to 2.4 and 4.9 f-stops at a high quality level.

Table 2 Dynamic range presented here is computed for noise thresholds of 1.0 (low image quality)

\begin{tabular}{|l|c|c|c|c|}
\hline Camera & $\begin{array}{c}\text { Dynamic } \\
\text { Range } \\
(\text { Low })\end{array}$ & $\begin{array}{c}\text { Dynamic } \\
\text { Range } \\
\text { (Medium) }\end{array}$ & $\begin{array}{c}\text { Dynamic } \\
\text { Range } \\
(\text { High })\end{array}$ & $\begin{array}{c}\text { Steps } \\
\text { detected }\end{array}$ \\
\hline Device A & 5.9 & 5.8 & 3.9 & 17 \\
\hline Device B & 6.1 & 5.8 & 3.5 & 18 \\
\hline Device C & 6.1 & 5.9 & 4.9 & 19 \\
\hline Device D & 6.2 & 5.8 & 2.4 & 19 \\
\hline Device E & 4.8 & 4.8 & 3.4 & 15 \\
\hline Device F & 5.5 & 5.5 & 3.2 & 17 \\
\hline Device R & 6.3 & & 5.3 & 19 \\
\hline
\end{tabular}

\subsection{Analysis of Tone Mapped Images and Effective Dynamic Range}

This section of the paper will present results obtained after tone mapping where a global and local operator was utilized. They are included in commercially available software for HDR imaging. Fig. 4 shows a gradation of recorded full range of intensities among devices. In the case of the total DR for a single shot, the results are consistent with the previously determined DR of MD cameras. Also, both HDR images generated with TMOs show no significant difference in extending the DR of images, nor there are significant differences recorded between devices. Local TMO did, however, influence the DR slightly. The analysis of the EDR after utilizing TMOs presented us with different results (Fig.5). As expected, the useful range of density values is lower for all images.

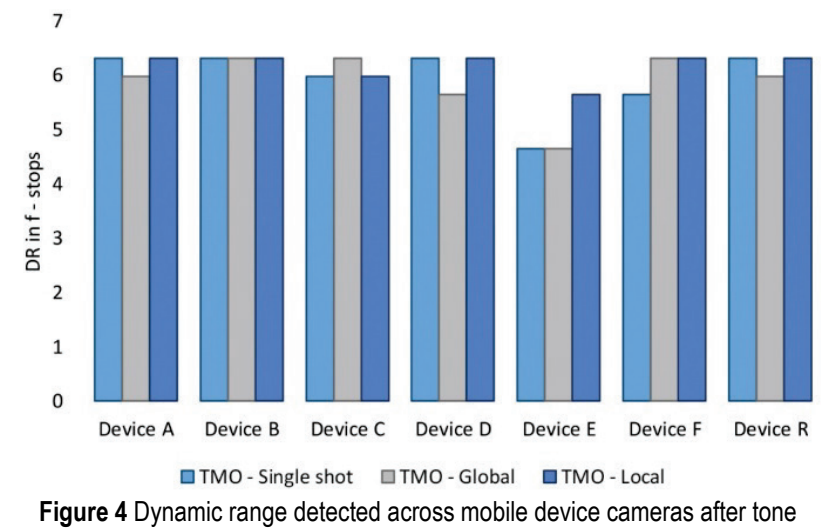

Figure 4 Dynamic range detected across mobile device cameras after tone mapping and compared to a single shot image

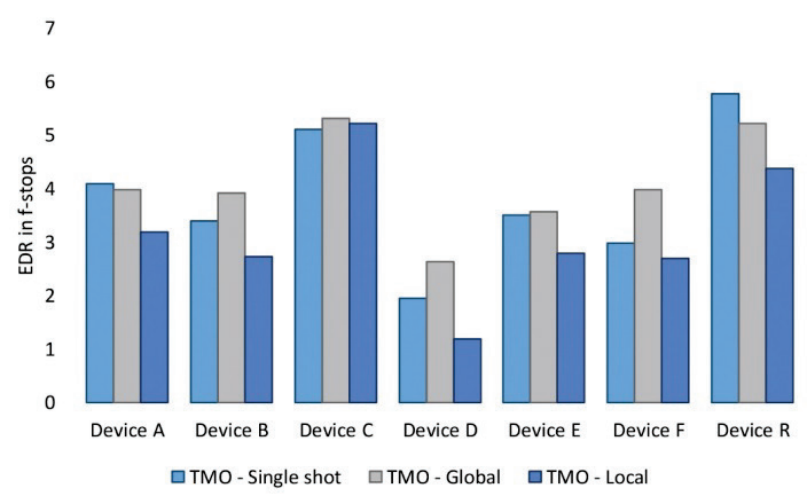

Figure 5 Effective dynamic range detected across mobile device cameras after tone mapping and compared to a single shot image

Global TMO extended the EDR for all images obtained from MD cameras. The highest recorded EDR of 5.31 f-stops with the global operator was for the image obtained with Device C, whereas the lowest quality level of the DR of $2.63 f$-stops presented Device D. Local TMO shows a decrease of the EDR for all images in comparison to a single shot. The lowest quality of 1.18 f-stops showed the image from Device C. Fig. 5 shows that EDR was extended when global TMO was used. It can be noticed how local TMO decreased EDR, for some devices, to a greater extent e.g. Device D. The extension of EDR was expected, as it was proposed by Robertson et al. [6], due to the fact that HDR image contains information in both low and high areas from data from each of the input images. Global TMOs are built upon simplicity and computational 
efficiency where the same mapping function is used for the whole image thus preserving the image signal but failing to keep the appropriate local contrast. This would explain the increase of the EDR for MD cameras as well. Local TMOs are in control of the local contrast where the image is perceived as more dramatic due to "halos" [23], and the decrease of useful intensities is expected.

\subsection{Noise Measurements}

Imatest has several types of noise calculations. To complement the analysis of our study, we discuss noise measured in pixel levels normalized to the difference in pixel levels for a density range of 1.5 and noise in $f$-stops with emphasis on high quality level. For noise measured in pixel levels, a single number is obtained which is used to characterize overall noise performance - the average Luminance channel noise (Tab. 3).

\begin{tabular}{|l|c|c|c|}
\multicolumn{1}{|c}{ Table 3 Values for noise thresholds of HDR images } \\
\begin{tabular}{|l|c|c|c|}
\hline Camera & $\begin{array}{c}\text { HDR image } \\
\text { Global TMO }\end{array}$ & $\begin{array}{c}\text { HDR image } \\
\text { Local TMO }\end{array}$ & Single image \\
\hline $\begin{array}{c}\text { Luminance } \\
\text { channel noise (\%) }\end{array}$ & $\begin{array}{c}\text { Average } \\
\text { Luminance } \\
\text { channel noise (\%) }\end{array}$ & $\begin{array}{c}\text { Average } \\
\text { Luminance } \\
\text { channel noise (\%) }\end{array}$ \\
\hline Device A & 0.9 & 2.09 & 1.18 \\
\hline Device B & 1.66 & 2.53 & 1.28 \\
\hline Device C & 0.58 & 1.2 & 0.71 \\
\hline Device D & 2.09 & 5.38 & 3.16 \\
\hline Device E & 0.91 & 1.9 & 0.88 \\
\hline Device F & 0.71 & 4.32 & 0.94 \\
\hline
\end{tabular}
\end{tabular}

It is noticeable that noise corresponds well to the trend of the EDR, as expected. Observably, Device C generated the lowest noise level of $0.71 \%$ for a single image and Device D the highest 3.16\%. When global TMO was applied it was noticeable how the level of noise decreased. On the other hand, the local TMO increased the level of noise, especially for Device D 5.38\% and Device F 4.32\% corresponding to poor image quality (Fig. 5).

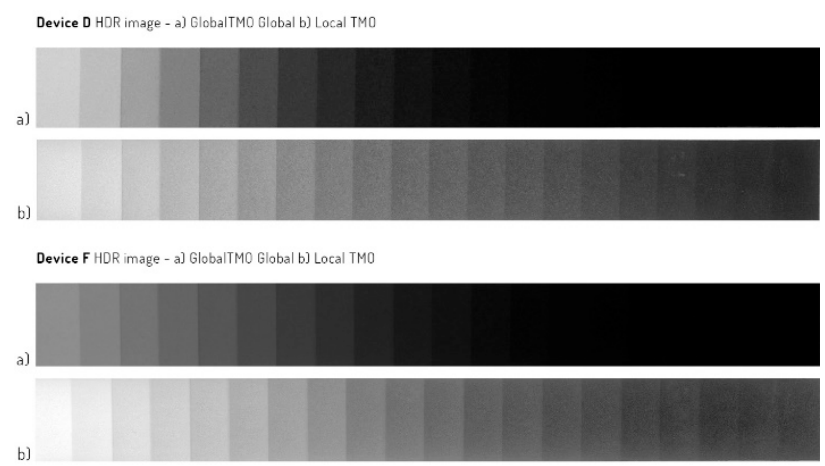

Figure 6 Display of photographed test chart with emphasis to noise deterioration between global and local TMOs

Comparatively, the level of noise in images with global and local TMO applied can be observed against the single shot image as shown in Fig. 7. The noise in these plots is scaled to the difference in pixel levels between $f$-stops which decreases as brightness decreases, that is, the darkest levels have the highest noise. It can be observed how the noise gradation is constant across channels for a single image and HDR image with local TMO applied. HDR image with global TMO shows lower noise threshold overall, however, the blue channel seems to have increased noise for some devices. This difference in pixel levels between $f$-stops is the inverse of the signal-to-noise ratio $(\mathrm{N} / \mathrm{S})$, marked as SNR_f in Imatest. These particular noise measurements were analysed because they correspond to HVS and they present an important value when calculating the EDR.
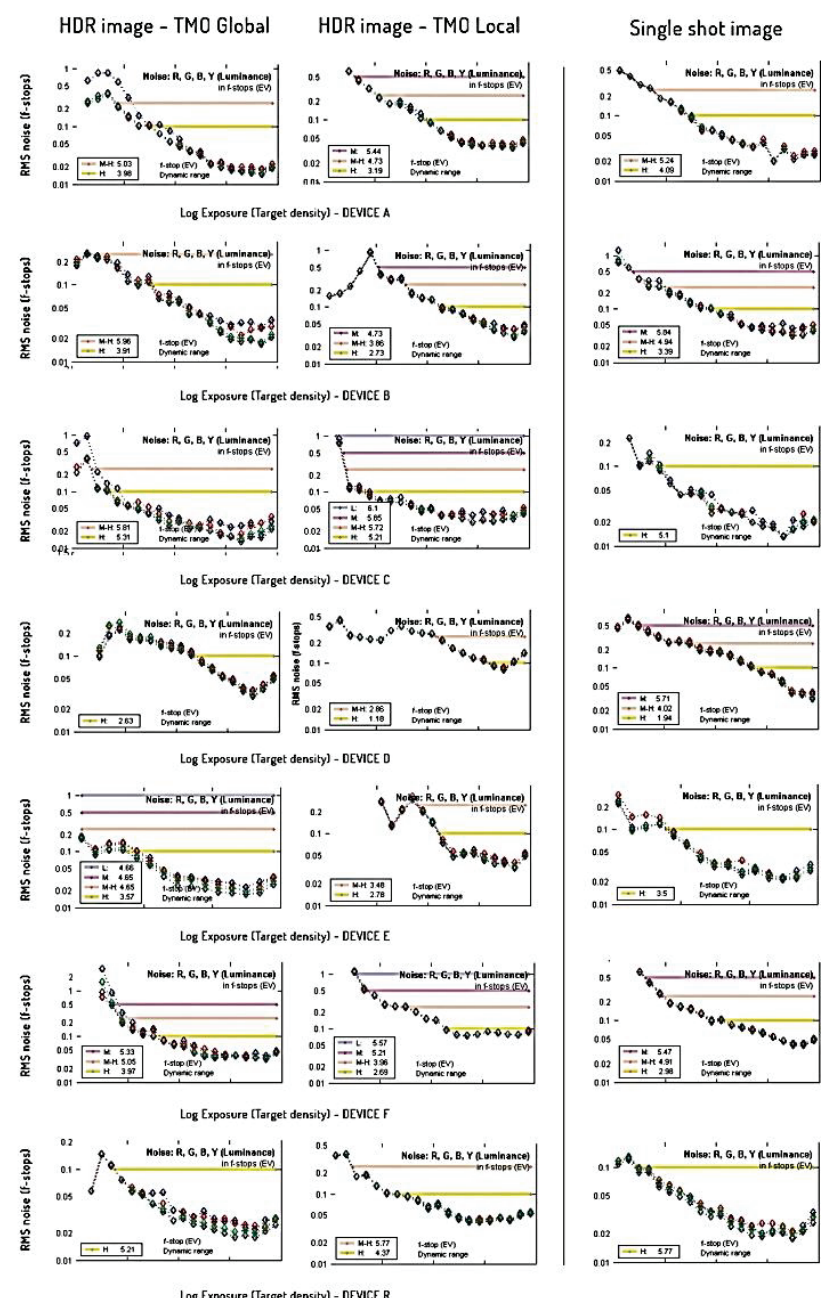

Figure 7 Plots from Imatest that show noise in $f$-stops (scene-referenced) Signal-to-Noise (SNR $f=1 / f$-stop noise). On the far right is the noise level of a single image. The bottom row presents referenced measurements obtained by DSLR camera. Noise thresholds of 0.1 (high image quality) are marked with yellow

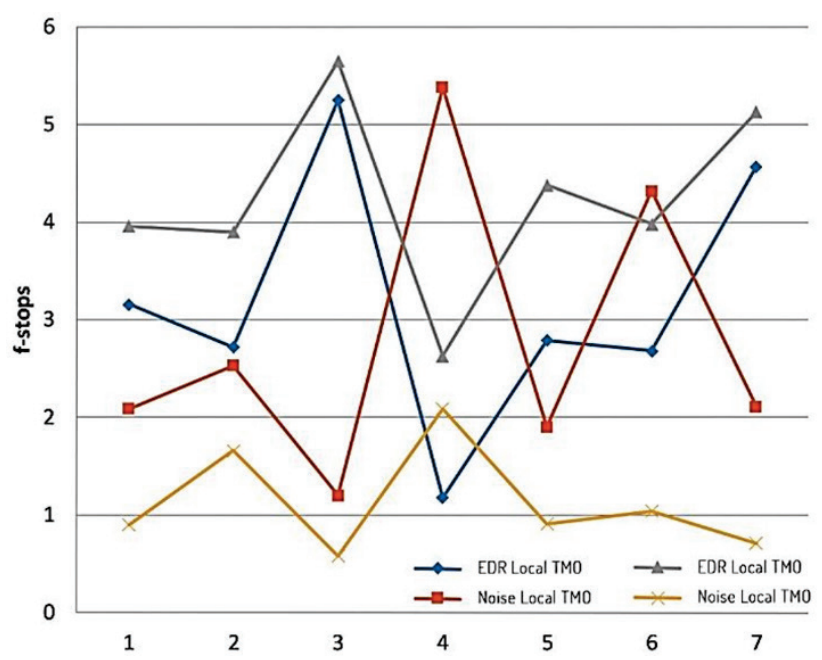

Figure 8 Graphic display of the relationship between noise and effective dynamic range for HDR images after tone mapping operators were applied 
Analysis of the relationship between noise level and EDR suggests the noise increase when there is lower range of usable intensities (Fig. 8). Device D and F show the lowest EDR. With the application of local operator this range decreases additionally leaving, for example, Device $\mathrm{D}$ with EDR of $1.18 f$-stops. This is partially due to the higher resolution of these two cameras (see Tab. 1).

\section{CONCLUSION}

Mobile devices are extensively used to acquire images on a daily basis. Built-in cameras in such devices maintain the same workflow and signal processing as DSLR cameras. However, the full range of useful intensities is encoded to some disadvantage of the hardware and software limitations. In this paper, we set out to evaluate digital still images acquired via MD cameras from the perspective of the effective dynamic range. To complement this evaluation even further, we applied a global and local TMO to understand how different procession of the operators influences encoded dynamic range. The results have shown that the automatic conversion function increased the digital output.

Additionally, greater nonlinearity among MD cameras was observed. The analysis of the tone mapped images revealed that MD cameras are not consistent in capturing the contrast present in the original HDR scene. Tone mapping with a global operator extended the effective dynamic range while the local tone mapping operator decreased the range and increased the noise level. While the resolution of MD cameras is perceived to be adequate for small screen display, a quality parameter such as dynamic range and noise which are the result of our evaluation suggest the low overall quality of the digital image generated by MD cameras. Namely, our results show that MD cameras show less reliable results in signal encoding in comparison to DSLR and great variations among different manufacturers. We can conclude that the effective dynamic range can be extended but only to a certain degree. Detected noise thresholds which correlate to low image quality are below the satisfactory quality level for a more serious application where image accuracy is highly important in shooting on the go cases. Hopefully, the results of this study provided an insightful evaluation of mobile device camera's characteristics which can aid further research in overcoming the limitations of smallsized digital image capture systems.

\section{Acknowledgements}

The authors wish to thank Radovan Slavuj for his valuable help in revising this paper. This study was supported by the Serbian Ministry of Science and Technological Development, Grant No. 35027.

\section{CONCLUSION}

[1] Jack, T. \& Holly, R. (1993). Tone Reproduction for Realistic Images. IEEE Computer Graphics and Applications, 13(6), 42-48. https://doi.org/10.1109/38.252554

[2] Koh, C. C., Mukherjee, J., \& Mitra, S. K. (2003). New efficient methods of image compression in digital cameras with color filter array. IEEE Transactions on Consumer Electronics, 49(4), 1448-1456.
https://doi.org/10.1109/TCE.2003.1261253

[3] Larson, G. W. \& Shakespeare, R. (2004). Rendering with Radiance: the art and science of lighting visualization, BookSurge LLC.

[4] Matkovic, K., Neumann, L., \& Purgathofer, W. (1997). A survey of tone mapping techniques. In $13^{\text {th }}$ Spring Conference on Computer Graphics, W. Straßer Ed., 163170.

[5] McNamara, A. (2001). Visual perception in realistic image synthesis. Computer Graphics Forum, 20(4), 211-224. https://doi.org/10.1111/1467-8659.00550

[6] Robertson, M. A., Borman, S., \& Stevenson, R. L. (2003). Estimation-theoretic approach to dynamic range enhancement using multiple exposures. Journal of Electronic Imaging, 12, 219-228. https://doi.org/10.1117/1.1557695

[7] Cai, Y. (2010). Mobile intelligence. Journal of Universal Computer Science, 16(12), 1650-1665.

[8] Rashvand, H. F. \& Hsiao, K. F. (2013). Smartphone intelligent applications: a brief review. Multimedia Systems, 21(1), 103-119. https://doi.org/10.1007/s00530-013-0335-z

[9] Urbano, C., Magalhães, L., Moura, J., Bessa, M., Marcos, A., \& Chalmers, A. (2010). Tone mapping operators on small screen devices: An evaluation study. Computer Graphics Forum, 29(8), 2469-2478. https://doi.org/10.1111/j.1467-8659.2010.01758.x

[10] Sharma, G. (2003). Digital Color Imaging Handbook, CRC Press LLC.

[11] Allen, E. \& Triantaphillidou, S. (2011). The manual of photography, Taylor \& Francis. https://doi.org/10.4324/9780080926803

[12] Wüller, D. (2006). Proposal for a Standard Procedure to Test Mobile Phone Cameras. Proc. of SPIE-IS\&T Electronic Imaging, 6069, 1-13. https://doi.org/10.1117/12.643739

[13] Burns, P. D. (2005). Tone-transfer (OECF) characteristics and spatial frequency response measurements for digital cameras and scanners. In Proceedings of SPIE, Vol. 5668, 123-128. https://doi.org/10.1117/12.585884

[14] ISO 14524:2009 Photography -- Electronic still-picture cameras -- Methods for measuring opto-electronic conversion functions, (OECFs), 2015.

[15] Hsu, W-F., Chuang, K. W., \& Hsu, Y. C. (2000). Comparisons of the camera OECF, the ISO speed, and the SFR of digital still-picture cameras. In Photonics Taiwan, International Society for Optics and Photonics, 104-111. https://doi.org/10.1117/12.389434

[16] Wüller, D. \& Gabele, H. (2007). The usage of digital cameras as luminance meters. Electronic Imaging Conference, 6502, 1-11, https://doi.org/10.1117/12.703205

[17] Hertel, D. W. \& Chang, E. (2007). OECF characterization of a non-linear HDR Color Camera for Automotive Applications. In Color and Imaging Conference, Society for Imaging Science and Technology, Vol. 2007, 244-248.

[18] Debevec, P. E. \& Malik, J. (2008). Recovering High Dynamic Range Radiance Maps from Photographs. In Proceedings SIGGRAPH '08, ACM New York, NY, USA. https://doi.org/10.1145/1401132.1401174

[19] Xiao, F., DiCarlo, J. M., Catrysse, P. B., \& Wandell, B. A. (2002). High dynamic range imaging of natural scenes. In Tenth Color Imaging Conference: Color Science, Systems, and Applications, Society for Imaging Science and Technology, Vol. 2002, 337-342.

[20] Nayar, S. K. \& Mitsunaga, T. (2002). High dynamic range imaging: spatially varying pixel exposures. Proceedings IEEE Conference on Computer Vision and Pattern Recognition. CVPR 2000 (Cat. No. PR00662), 1, 472-479, https://doi.org/10.1109/CVPR.2000.855857

[21] Chiu, K., Herf, M., Shirley, P. Swamy, S., Wang, C., \& Zimmerman, K. (1993). Spatially Nonuniform Scaling 
Functions for High Contrast Images. Proceedings of Graphics Interface, 245-253. https://doi.org/10.1.1.136.5090

[22] Fattal, R., Lischinski, D., \& Werman, M. (2002). Gradient Domain High Dynamic Range Compression. ACM Transactions on Graphics (TOG), 21(3), 246-256. https://doi.org/10.1145/566654.566573

[23] Durand, F. \& Dorsey, J. (2002). Fast bilateral filtering for the display of high-dynamic-range images. ACM Transactions on Graphics, 21, 257-266. https://doi.org/10.1145/566654.566574

[24] Ashikhmin, M. (2002). A tone mapping algorithm for high contrast images. Proceedings of the $13^{\text {th }}$ Eurographics workshop on Rendering, Eurographics Association, 145-156

[25] Reinhard, E., Stark, M., Shirley, P., \& Ferwerda, J. (1998). Photographic Tone Reproduction for Digital Images. ACM Transactions on Graphics (TOG), 21(3), 267-276. https://doi.org/10.1145/566654.566575

[26] Larson, G. W., Rushmeier, H., \& Piatko, C. (1997). A visibility matching tone reproduction operator for high dynamic range scenes. Visualization and Computer Graphics, IEEE Transactions on, 3(4), 291-306. https://doi.org/10.1109/2945.646233

[27] Pattanaik, S. N., Ferwerda, J. A., Fairchild, M. D., \& Greenberg, D. P. (1998). A multiscale model of adaptation and spatial vision for realistic image display. In Proceedings of the $25^{\text {th }}$ annual conference on Computer graphics and interactive techniques, ACM, 287-298. https://doi.org/10.1145/280814.280922

[28] Kuang, J., Yamaguchi, H., Johnson, G. M., \& Fairchild, M. D. (2004). Testing HDR Image Rendering Algorithms. Proceedings IS\&T/SID Color Imaging Conference, 315-320.

[29] Drago, F., Myszkowski, K., Annen, T., \& Chiba, N. (2003). Adaptive logarithmic mapping for displaying high contrast scenes. Computer Graphics Forum, 22(3), 419-426. https://doi.org/10.1111/1467-8659.00689

[30] Narwaria, M., Perreira Da Silva, M., Le Callet, P., \& Pepion, R. (2014). Tone mapping based HDR compression: Does it affect visual experience? Signal Processing: Image Communication, 29(2), 257-273. https://doi.org/10.1016/j.image.2013.09.005

[31] Sencar, H. T. \& Memon, N. (2012). Digital image forensics: There is more to a picture than meets the eye, Springer Science \& Business Media.

[32] Mantiuk, R., Cichowicz, M., Smyk, M. (2012). Implementation of HDR photographic pipeline in mobile devices. In Image Analysis and Recognition, Springer Berlin Heidelberg, 367-374. https://doi.org/10.1007/978-3-642-31295-3_43

[33] Čadík, M., Wimmer, M., Neumann, L., \& Artusi, A. (2008). Evaluation of HDR tone mapping methods using essential perceptual attributes. Computers and Graphics (Pergamon), 32(3), 330-349. https://doi.org/10.1016/j.cag.2008.04.003

[34] Ledda, P., Chalmers, A., Troscianko, T., \& Seetzen, H. (2005). Evaluation of tone mapping operators using a High Dynamic Range display. ACM Transactions on Graphics, 24(3), 640-648. https://doi.org/10.1145/1073204.1073242

[35] Yoshida, A., Blanz, V., Myszkowski, K., \& Seidel, H-P. (2005). Perceptual evaluation of tone mapping operators with real-world scenes. Electronic Imaging, International Society for Optics and Photonics. https://doi.org/10.1117/12.587782

[36] Melo, M., Bessa, M., Barbosa, L., Debattista, K., Chalmers, A. (2015). Screen reflections impact on HDR video tone mapping for mobile devices: an evaluation study. EURASIP Journal on Image and Video Processing, 2015(1), 1-13. https://doi.org/10.1186/s13640-015-0094-1

[37] Hanhart, P., Bernardo, M. V., Pereira, M., Pinheiro, A. M. G., Ebrahimi, T. (2015). Benchmarking of objective quality metrics for HDR image quality assessment. EURASIP Journal on Image and Video Processing, 2015(1), 39. https://doi.org/10.1186/s13640-015-0091-4

[38] Koren, N. (2006). The Imatest program: comparing cameras with different amounts of sharpening. In Proc. SPIE 6069, Digital Photography II, 60690L, International Society for Optics and Photonics. https://doi.org/10.1117/12.650848

[39] Imatest, "Stepchart". (2004). http://www.imatest.com/ support/modules/stepchart/. (Accessed: 01-Jan-2015).

\section{Contact information:}

Irma PUŠKAREVIĆ, PhD

University of Novi Sad, Faculty of Technical Sciences, Department of Graphic Engineering and Design Trg Dositeja Obradovića 6, 21000 Novi Sad, Serbia Phone: +38121 4852628

E-mail: irma@uns.ac.rs

\section{Uroš NEDELJKOVIĆ, Assoc. Prof. PhD}

University of Novi Sad, Faculty of Technical Sciences, Department of Graphic Engineering and Design Trg Dositeja Obradovića 6, 21000 Novi Sad, Serbia Phone: +38121 4852624

E-mail: urosned@uns.ac.rs

Dragoljub NOVAKOVIĆ, Prof. PhD

University of Novi Sad, Faculty of Technical Sciences, Department of Graphic Engineering and Design Trg Dositeja Obradovića 6, 21000 Novi Sad, Serbia Phone: +38121 4852620

E-mail: novakd@uns.ac.rs

Ivan PINĆJER, Asst. Prof. PhD

University of Novi Sad, Faculty of Technical Sciences,

Department of Graphic Engineering and Design

Trg Dositeja Obradovića 6, 21000 Novi Sad, Serbia

Phone: +38121 4852624

E-mail: pintier@uns.ac.rs 